University of Texas at El Paso

ScholarWorks@UTEP

$7-2020$

\title{
Grading Homeworks, Verifying Code: How Thorough Should the Feedback Be?
}

Francisco Zapata

The University of Texas at El Paso, fcozpt@outlook.com

Olga Kosheleva

The University of Texas at El Paso, olgak@utep.edu

Vladik Kreinovich

The University of Texas at El Paso, vladik@utep.edu

Follow this and additional works at: https://scholarworks.utep.edu/cs_techrep

Part of the Applied Mathematics Commons, and the Computer Sciences Commons Comments:

Technical Report: UTEP-CS-20-86

\section{Recommended Citation}

Zapata, Francisco; Kosheleva, Olga; and Kreinovich, Vladik, "Grading Homeworks, Verifying Code: How Thorough Should the Feedback Be?" (2020). Departmental Technical Reports (CS). 1478.

https://scholarworks.utep.edu/cs_techrep/1478

This Article is brought to you for free and open access by the Computer Science at ScholarWorks@UTEP. It has been accepted for inclusion in Departmental Technical Reports (CS) by an authorized administrator of ScholarWorks@UTEP.For more information, please contact Iweber@utep.edu. 


\title{
Grading Homeworks, Verifying Code: How Thorough Should the Feedback Be?
}

\author{
Francisco Zapata, Olga Kosheleva, and Vladik Kreinovich \\ University of Texas at El Paso \\ $500 \mathrm{~W}$. University \\ El Paso, TX 79968, USA \\ fcozpt@outlook.com, olgak@utep.edu,vladik@utep.edu
}

\begin{abstract}
In the ideal world, we should assign many homeworks and give a thorough feedback for each homework. However, in reality, the instructor's time is limited, so we can either assign few homeworks and give a detailed feed back for all of them, or we can assign many homeworks, but give a less thorough feedback. What is the optimal thoroughness? A similar question can be raised for code verification: what is the optimal amount of feedback that should be provided to each programmer? In this paper, we provide answers to these questions.
\end{abstract}

\section{Formulation of the Problem}

How thoroughly should we grade the homeworks? In the ideal world, we should assign many homeworks, and grade each of them very thoroughly. In reality, our time - and if we have a Teaching Assistant for the class, the time of the Teaching Assistant - is limited. So, we have a choice:

- we can assign few homeworks and grade all of them very thoroughly,

- or we can assign many homeworks and grade all of them less thoroughly.

What is the optimal level of thoroughness?

Comment. Doing homeworks helps students, even if these homeworks are not graded at all - since this provides the students with additional practice. These are many ways to entice the students to do homeworks even when not all homeworks will be graded:

- we can assign several problems, but grade only some of them; the selection of graded problems can be decided by a random choice after the homeworks are submitted, so the students do not know beforehand which problems will be graded; 
- another idea is, after the homework is submitted, to randomly select students whose homeworks will be graded.

It is also possible to have a combination of these two ideas.

But no matter what we do, the same question remains: how thoroughly should we grade the homeworks?

How thoroughly should we verify the code. Usually, when a company designs software, the resulting code is not only tested to make sure it works it is also verified by specialists who make sure that this code is well-written. Again, in the ideal world, every line of code should be thoroughly analyzed and verified, and the detailed feedback should be provided to the programmer. In practice, the verifier's time is limited, so we have a choice:

- we can verify fewer lines of code and provide more thorough feedback,

- or we can verify more lines of code and provide less thorough feedback.

What is the optimal level of thoroughness?

What we do in this paper. In this paper, we use the general decision techniques to come up with the optimal levels of thoroughness.

At first, we consider a simplified model in which we ignore the difference between different students (or programmers). Then, we consider a more realistic model, in which we take the difference between students (or programmers) into account.

Comment. In this paper, for clarity, all the formulas are explained on the example of teaching, when we decide on the amount of feedback provided to each homework. However, the exact same formulas and conclusions are also valid for code verification, when we have to decide on the optimal amount of feedback provided to each programmer.

\section{Simplified Case, When We Ignore the Differ- ence Between Students}

Description of the simplified case. In this section, we consider the simplified case, when we ignore the difference between students, and assume that the effect of feedback is the same for all students.

Analysis of the problem. The more time we spend on feedback, the more thorough this feedback, the larger the effect it has on the person who receives this feedback - be it a student or a programmer.

Usually, very little feedback is practically not helpful at all, then we have an increase, but after that, too much details will be mostly wasted - this is like micromanagement.

Notations. To describe the resulting problem in precise terms, let us introduce the following notations: 
- Let us denote the effect of a piece of feedback that took $t$ hours by $f(t)$.

- Let $T$ denote the overall time allocated to producing this feedback.

Let us formulate the problem in precise terms. What we want is find the value $n$ and the values $t_{1}, \ldots, t_{n}$ for which

$$
t_{1}+\ldots+t_{n}=T,
$$

and for which, among all such tuples, the overall effect

$$
E=f\left(t_{1}\right)+\ldots+f\left(t_{n}\right)
$$

is the largest possible.

Towards solving the problem. Let us use the usual Lagrange multiplier technique to reduce the constrained optimization problem (1)-(2) to the unconstrained optimization problem of maximizing the following functional

$$
f\left(t_{1}\right)+\ldots+f\left(t_{n}\right)+\lambda \cdot\left(t_{1}+\ldots+t_{n}-T\right),
$$

where $\lambda$ is the Lagrange multiplier.

Differentiating the expression (3) with respect to each unknown $t_{i}$ and equating the derivative to 0 , we conclude that

$$
f^{\prime}\left(t_{i}\right)+\lambda=0,
$$

where $f^{\prime}(t)$ denotes the derivative of the function $f^{\prime}(t)$. Thus, for all $i$, we have the same value of the derivative $f^{\prime}\left(t_{i}\right)=-\lambda$. So, in general, the corresponding values of $t_{i}$ should be the same:

$$
t_{1}=\ldots=t_{n}
$$

Hence,

$$
E=f\left(t_{1}\right)+\ldots+f\left(t_{n}\right)=n \cdot f\left(t_{1}\right) .
$$

From the formula (1), we can similarly conclude that $n \cdot t_{1}=T$. Therefore,

$$
n=\frac{T}{t_{1}} .
$$

Substituting the expression (7) into the formula (6) for the overall effect, we conclude that

$$
E=\frac{T}{t_{1}} \cdot f\left(t_{1}\right) .
$$

Maximizing this expression is equivalent to maximizing the expression

$$
\frac{E}{T}=\frac{f\left(t_{1}\right)}{t_{1}} .
$$

Thus, we arrive at the following conclusion. 
Conclusion. The optimal time $t_{1}$ to spend on each feedback is the time that maximizes the ratio (9).

Comment. It is worth mentioning that the optimal time $t_{1}$ that needs to be spent on each feedback does not depend on the overall time $T$. So, if we have more time $T$ for grading (e.g., if we get an additional teaching assistant), we should assign more homeworks - but keep the same level of thoroughness as before.

Let us make this conclusion more detailed. When we have an analytical expression for $f(t)$, then the largest value of the expression (9) can be obtained if we differentiate the expression (9) with respect to $t_{1}$ and equate the derivative to 0 . As a result, we get the formula

$$
\frac{f^{\prime}\left(t_{1}\right) \cdot t_{1}-f\left(t_{1}\right)}{t_{1}^{2}}=0,
$$

i.e., equivalently,

$$
f^{\prime}\left(t_{1}\right) \cdot t_{1}=f\left(t_{1}\right)
$$

Conclusion in more detail. The optimal time $t_{1}$ to spend on each feedback is the time that satisfies the equation (11).

What is the empirical dependence $f(t)$. According to [2] (see also [1]), the effect of each stimulus - including the feedback - is proportional to

$$
f(t)=\frac{t^{q}}{t^{q}+c},
$$

for some empirical constants $q$ and $c$.

Let us show how to solve the equation (11) for this function $f(t)$.

Case of empirical dependence: analysis of the problem. The derivative $f^{\prime}(t)$ of the expression (12) is easy to find when we realize that

$$
f(t)=1-\frac{c}{t^{q}+c} .
$$

Differentiating the right-hand side of the formula (13), we conclude that

$$
f^{\prime}(t)=\frac{c \cdot q \cdot t^{q-1}}{\left(t^{q}+c\right)^{2}} .
$$

Thus, the equation (11) takes the form

$$
\frac{c \cdot q \cdot t_{1}^{q}}{\left(t_{1}^{q}+c\right)^{2}}=\frac{t_{1}^{q}}{t_{1}^{q}+c} .
$$

If we divide both side of this equality by $t_{1}^{q}$ and multiply both sides by $\left(t_{1}^{q}+c\right)^{2}$, we get the following equality:

$$
c \cdot q=t_{1}^{q}+c,
$$


hence

$$
t_{1}^{q}=c \cdot(q-1)
$$

So, we arrive at the following conclusion.

Case of empirical dependence: conclusion. The optimal time $t_{1}$ to spend on each feedback is equal to

$$
t_{1}=(c \cdot(q-1))^{1 / q},
$$

where $c$ and $q$ are the parameters of the dependence (12).

\section{General Case, When We Take Into Account That Students Are Different}

Description of the general case. Let us now consider the general case, when we take the difference between the students into account.

To be fair, we allocate equal time to each student.

\section{Notations.}

- Let $s$ denote the number of students.

- Let $f_{i}(t)$ denote the effect, on the $i$-th students, of the feedback that the instructor produced during time $t$.

Analysis of the problem: general dependence. In the fair approach, out of the total time $T$, the instructor allocates time $T / s$ to each student. Thus, what we want is find, for each student $i$, is the number of assignments $n_{i}$ and the values $t_{i, 1}, \ldots, t_{i, n_{i}}$ for which

$$
t_{i, 1}+\ldots+t_{i, n_{i}}=\frac{T}{s}
$$

and for which, among all such tuples, the overall effect

$$
E_{i}=f_{i}\left(t_{i, 1}\right)+\ldots+f_{i}\left(t_{i, n_{i}}\right)
$$

is the largest possible.

Similarly to the previous section, we conclude that all the times $t_{i 1}, \ldots, t_{i n_{i}}$ should be equal:

$$
t_{i, 1}=\ldots=t_{i, n_{i}}
$$

and the optimal value of $t_{i 1}$ is the one that maximizes the expression

$$
\frac{f_{i}\left(t_{i, 1}\right)}{t_{i, 1}}
$$

i.e., equivalently, for which the following equality holds:

$$
f_{i}^{\prime}\left(t_{i, 1}\right) \cdot t_{i, 1}=f_{i}\left(t_{i, 1}\right)
$$


General dependence: conclusion. The optimal time $t_{i, 1}$ allocated to provide feedback for each homework of $i$-th students should maximize the expression (22), or, equivalently, should satisfy the equation (23).

Analysis of the problem: case of empirical dependence. In particular, for the case when

$$
f_{i}(t)=\frac{t^{q_{i}}}{t^{q_{i}}+c_{i}}
$$

for some coefficients $q_{i}$ and $c_{i}$, the optimal time $t_{i, 1}$ has the form

$$
t_{i, 1}=\left(c_{i} \cdot\left(q_{i}-1\right)\right)^{1 / q_{i}} .
$$

Empirical dependence: conclusion. For the empirical dependence (24), the optimal time $t_{i, 1}$ allocated to provide feedback for each homework of the $i$-th student is determined by the formula (25).

Comment. Since we allocated the same time $T / s$ to each student, and the optimal times $t_{i, 1}$ are, in general, different for different students, this means that the number of homeworks

$$
n_{i}=\frac{T / s}{t_{i, 1}}
$$

assigned to different students should be, in general, different:

- to some students, we assign fewer homeworks, and provide more thorough feedback, while

- to other students, we assign more homeworks, and provide less thorough feedback.

\section{Acknowledgments}

This work was supported in part by the National Science Foundation grants 1623190 (A Model of Change for Preparing a New Generation for Professional Practice in Computer Science) and HRD-1242122 (Cyber-ShARE Center of Excellence).

\section{References}

[1] L. Bokati, V. Kreinovich, and Doan Thanh Ha, How the Proportion of People Who Agree to Perform a Task Depends on the Stimulus: A Theoretical Explanation of the Empirical Formula, University of Texas at El Paso, Department of Computer Science, Technical Report UTEP-CS-20-41, 2020, http://www.cs.utep.edu/vladik/2020/tr20-41.pdf 
[2] M. Khani, A. Ahmadi, and H. Hajary, "Distributed task allocation in multi-agent environments using cellular learning automata", Soft Computing, 2019, Vol. 23, pp. 1199-1218. 